\title{
Bubble-Pen Lithography
}

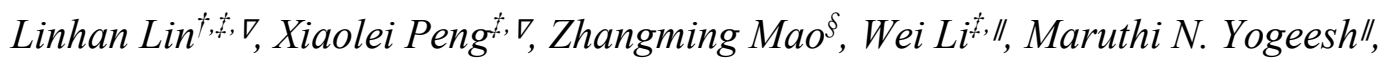

Bharath Bangalore Rajeeva ${ }^{\ddagger}$ Evan P. Perillo", Andrew K. Dunn ${ }^{\#}$, Deji Akinwande ${ }^{\ddagger}, \|$, and Yuebing Zheng ${ }^{\dagger, t, *}$

${ }^{\dagger}$ Department of Mechanical Engineering, The University of Texas at Austin, Austin, TX 78712, USA.

Materials Science \& Engineering Program and Texas Materials Institute, The University of Texas at Austin, Austin, TX 78712, USA.

${ }^{\S}$ Department of Engineering Science and Mechanics, The Pennsylvania State University, University Park, PA 16802, USA.

"Microelectronics Research Centre, The University of Texas at Austin, Texas 78758, USA.

\#Department of Biomedical Engineering, The University of Texas at Austin, Austin, TX, 78712, USA

จThese authors contributed equally to this work.

*e-mail: zheng@austin.utexas.edu 
a

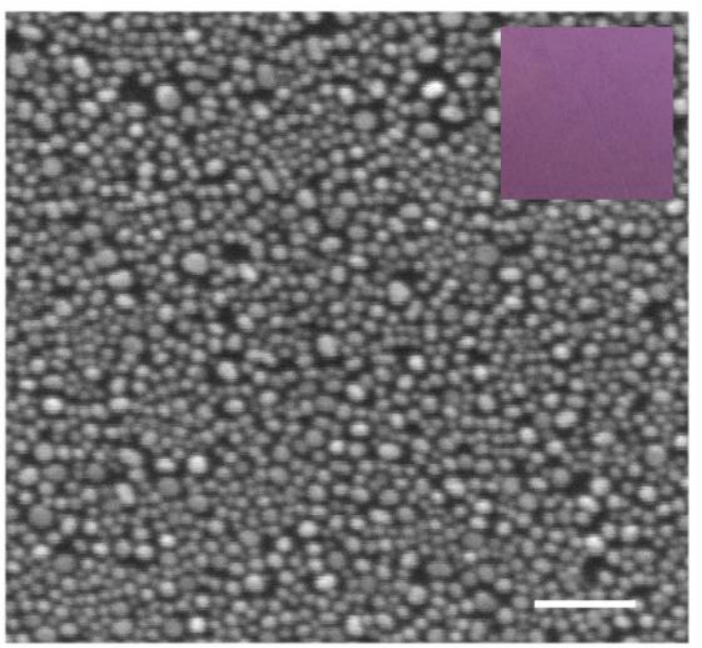

c

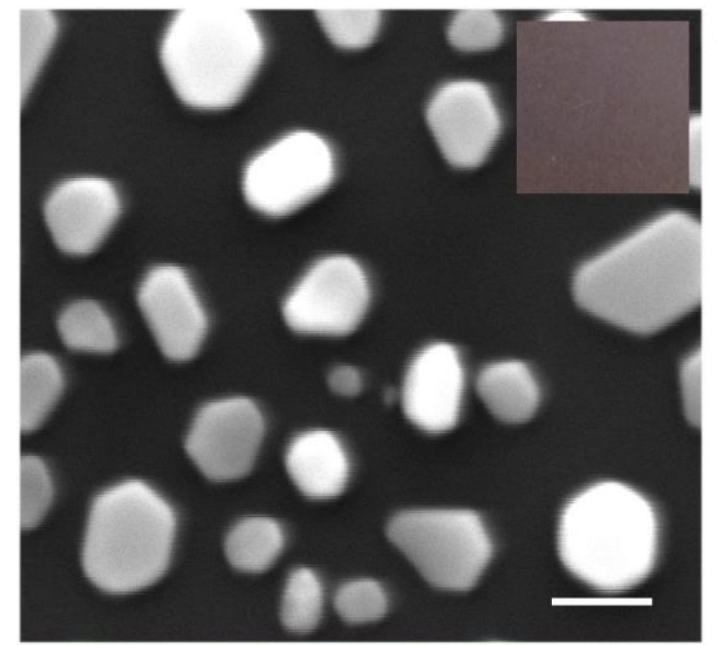

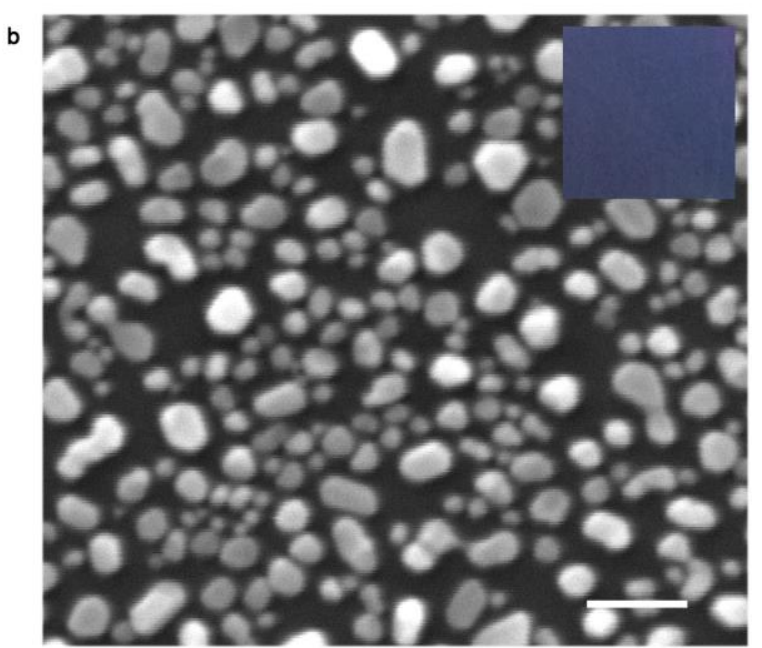

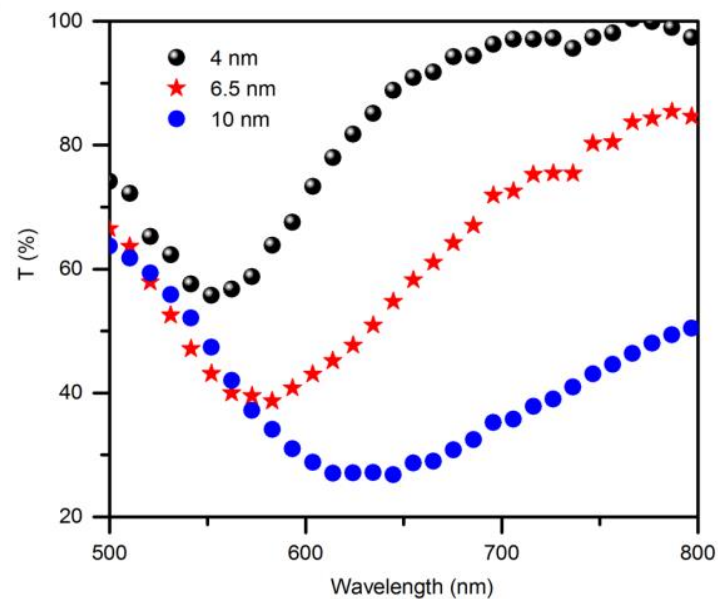

Figure S1. Optimization of the Au nanoislands (AuNIs) as the plasmonic substrate by tuning the pre-annealing thickness of $\mathrm{Au}$ thin films. Scanning electron micrographs of the AuNIs after thermal annealing at $550{ }^{\circ} \mathrm{C}$ for 2 hours with the initial thin film thickness of (a) $4 \mathrm{~nm}$, (b) $6.5 \mathrm{~nm}$, (c) $10 \mathrm{~nm}$. The insets show the optical images of the samples. (d) Absorption spectra of the AuNIs as shown in a-c. Scale bar: $200 \mathrm{~nm}$. 


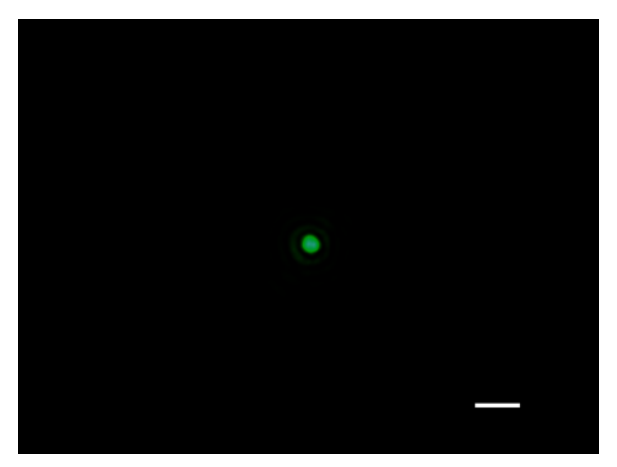

Figure S2. Optical image of a laser beam for the generation of a microbubble. The size of the laser beam is $2 \mu \mathrm{m}$. Scale bar: $5 \mu \mathrm{m}$.
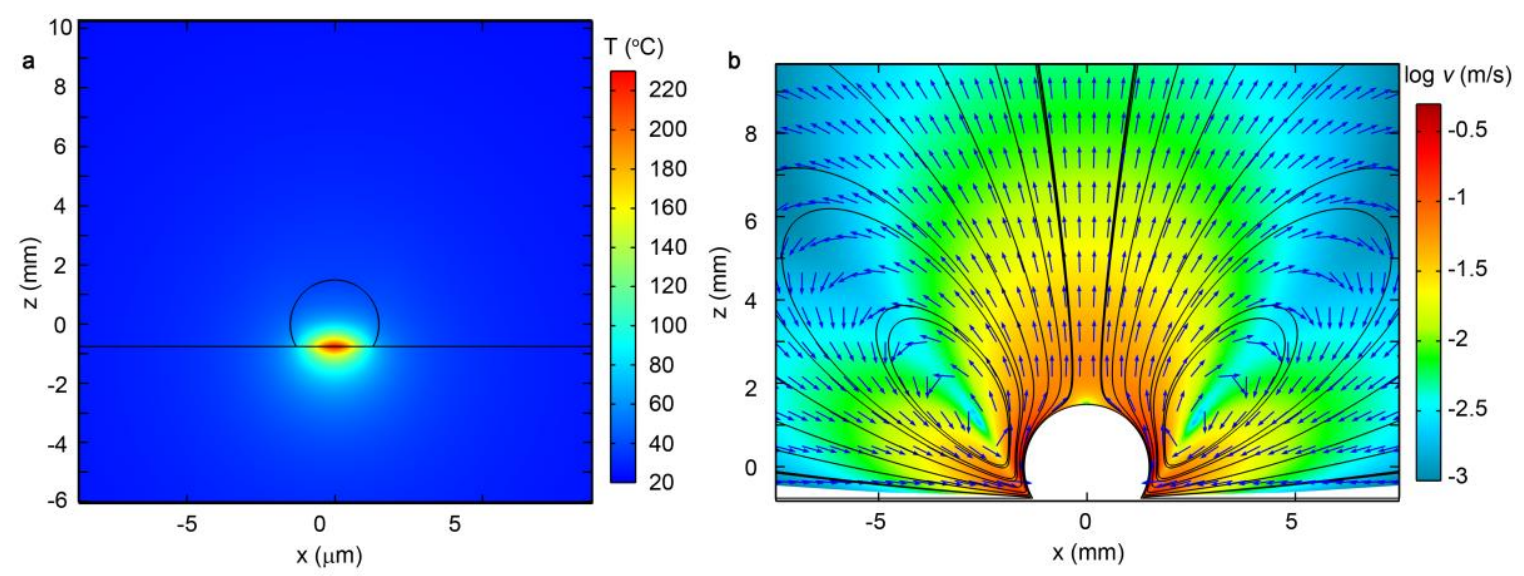

Figure S3. CFD simulations of a $3 \mu \mathrm{m}$ bubble. (a) Simulated temperature distribution around the microbubble. (b) Simulated flow velocity distribution around the microbubble with logarithmic scale. The laser power intensity is $0.97 \mathrm{~mW} / \mu \mathrm{m}^{2}$. The maximum temperature of $220{ }^{\circ} \mathrm{C}$ is at the center of the bubble/substrate interface. The minimum temperature around the bubble is $\sim 20^{\circ} \mathrm{C}$. A maximum flow velocity of $\sim 0.5 \mathrm{~m} / \mathrm{s}$ is generated around the bubble.
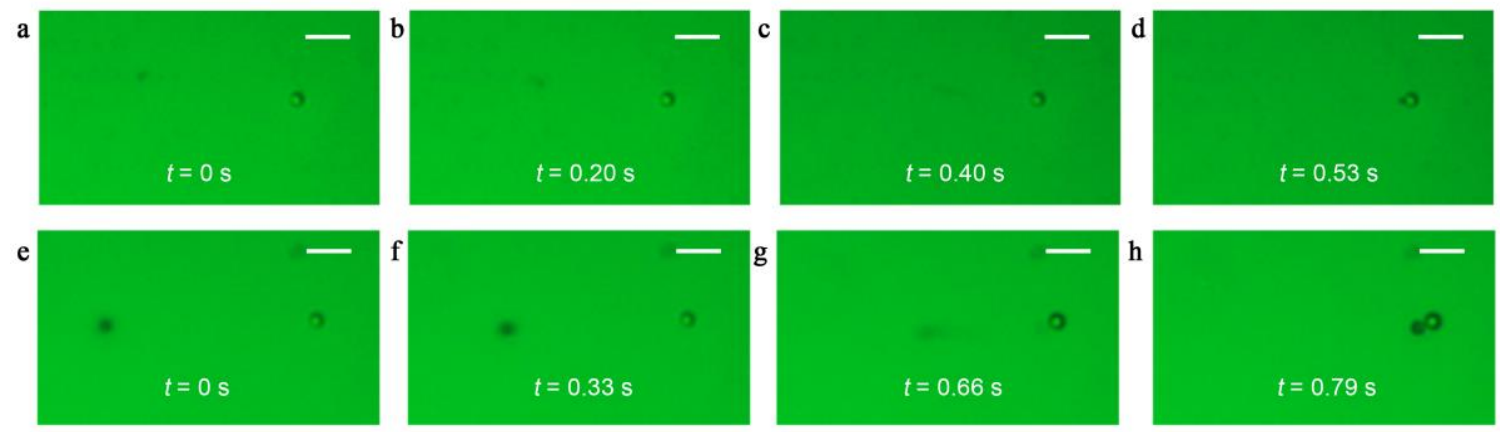
Figure S4. Time-resolved trapping processes of single PS beads by a microbubble. The sizes of the PS beads in (a)-(d) and (e)-(h) are $540 \mathrm{~nm}$ and, $0.96 \mu \mathrm{m}$, respectively. The diameter and power density of the laser beam are $2 \mu \mathrm{m}$ and $0.56 \mathrm{~mW} / \mu \mathrm{m}^{2}$. The average trapping speeds are estimated as 31.1 and $27.5 \mu \mathrm{m} / \mathrm{s}$ for the $540 \mathrm{~nm}$ and $0.96 \mu \mathrm{m}$ PS beads, respectively. Scale bar: 5 $\mu \mathrm{m}$.

a

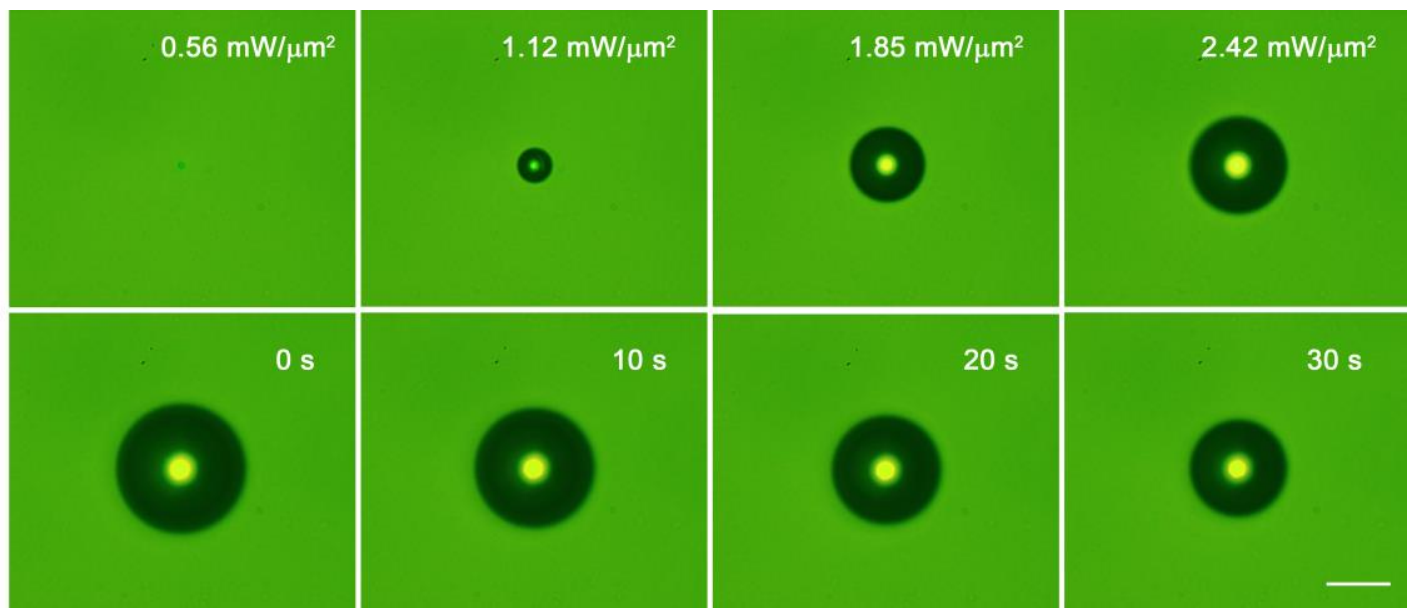

Figure S5. Bubble expansion and shrinkage process. (a) Bubble expansion with increased laser power densities. (b) Bubble shrinkage when the laser is off. Scale bar: $10 \mu \mathrm{m}$.

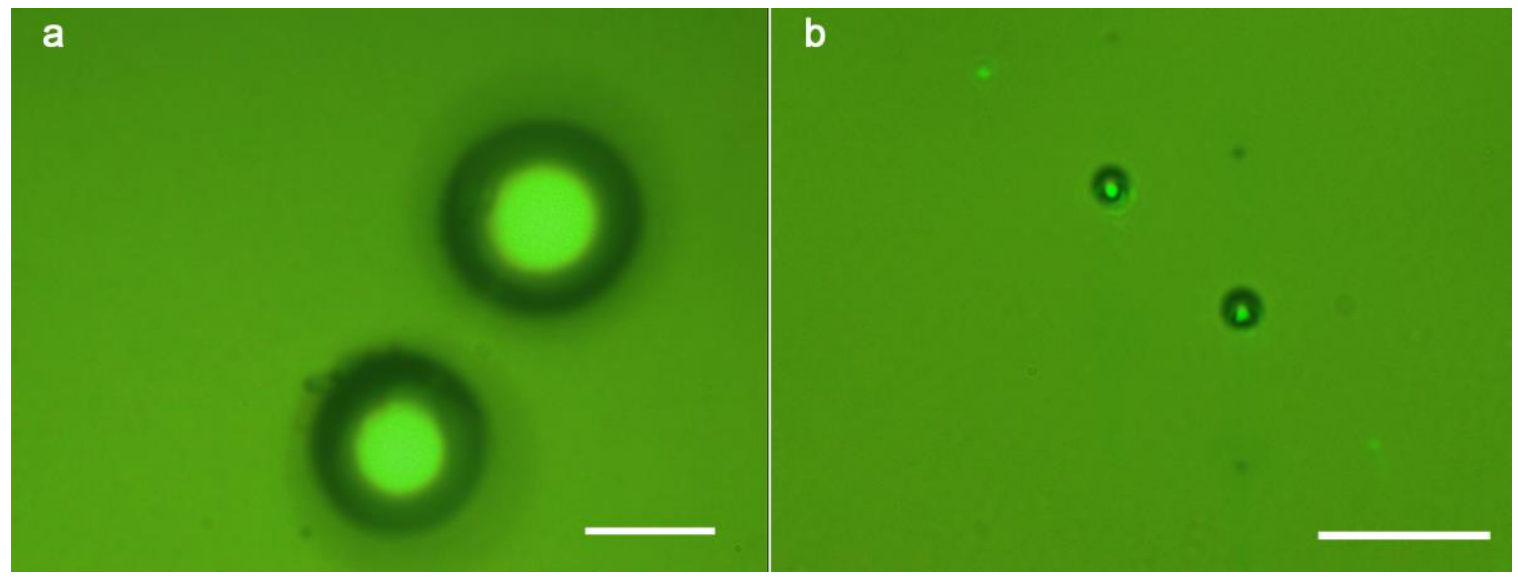

Figure S6. Generation of two microbubbles on the AuNIs substrate using the (a) digital micromirror device and (b) spatial light modulator, respectively. Scale bar: $10 \mu \mathrm{m}$. 

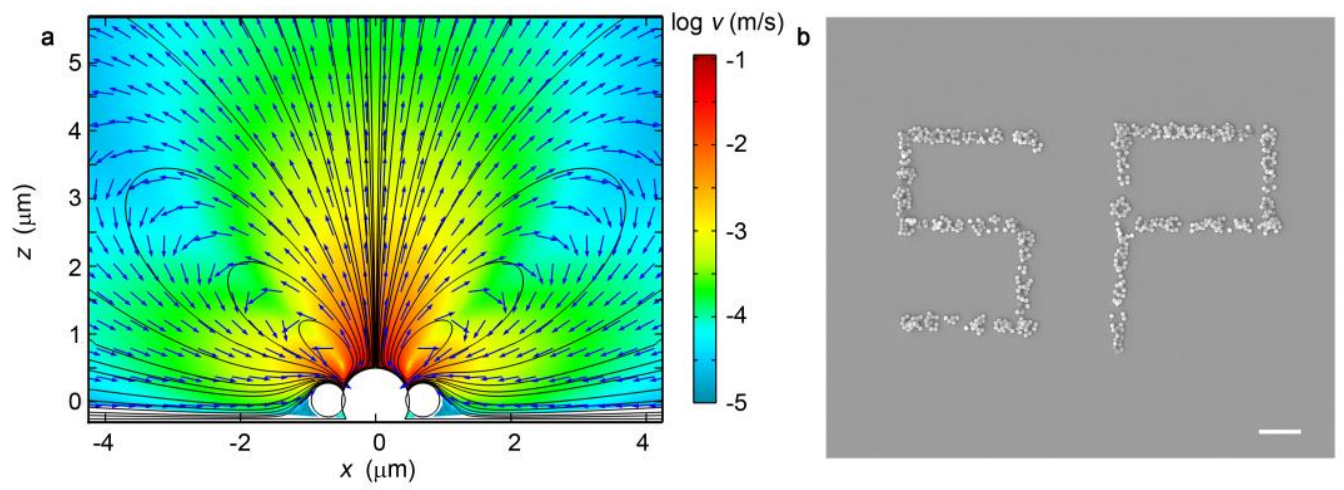

Figure S7. Writing "SP" pattern of $540 \mathrm{~nm}$ PS beads with $1 \mu \mathrm{m}$ bubbles. (a) CFD simulations of the flow velocity distribution around the $1 \mu \mathrm{m}$ microbubble when the $500 \mathrm{~nm}$ PS beads are trapped on the bubble surface. The trapping of the first layer of PS beads modifies the flow velocity distribution. The beads can be subsequently trapped either around or on the top of the first layer. The 3D hollow structure cannot be formed due to the limited bubble surface area. (b) Scanning electron micrograph of $540 \mathrm{~nm}$ PS beads in "SP" pattern, which corresponds to the dark-field optical image in Fig. 5b. Scale bar: $5 \mu \mathrm{m}$.

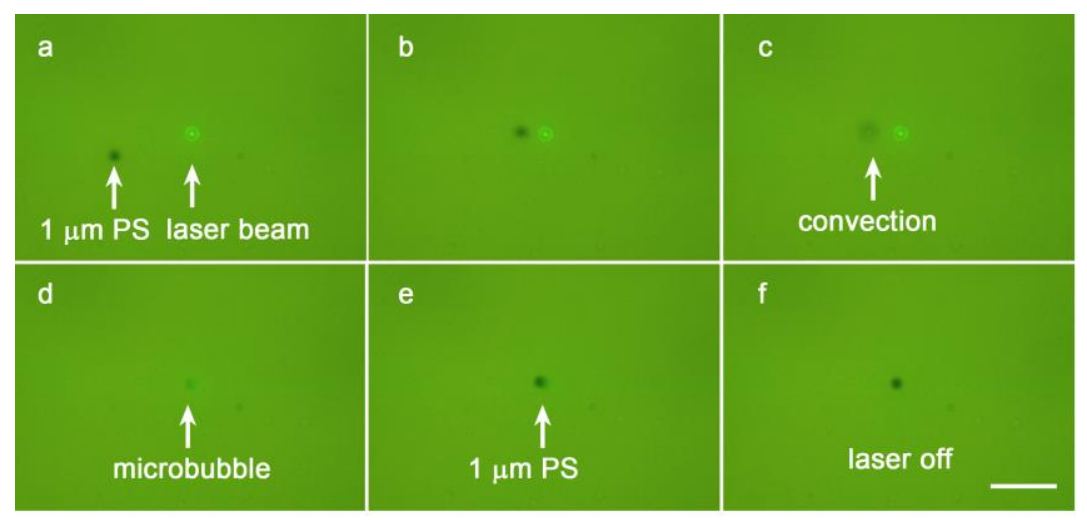

Figure S8. Different particle behaviors before and after generation of the microbubbles. (a) Laser beam on without generation of microbubble. (b) $0.96 \mu \mathrm{m}$ PS bead dragged toward the laser beam by natural convection. (c) PS bead move upward following the convective flow. (d) Generation of a microbubble. (e) A PS bead trapped and immobilized on the substrate. (f) PS bead patterned on the substrate when the laser is off. Scale bar: $10 \mu \mathrm{m}$. 


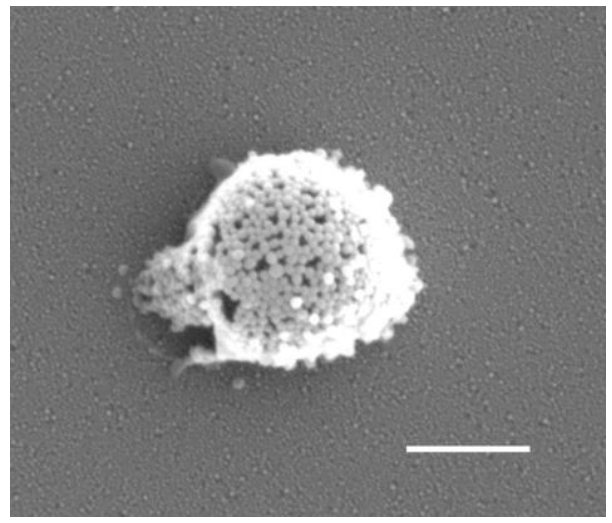

Figure S9. Scanning electron micrograph of the 3D hollow structure of $60 \mathrm{~nm}$ PS beads. Scale bar: $1 \mu \mathrm{m}$.
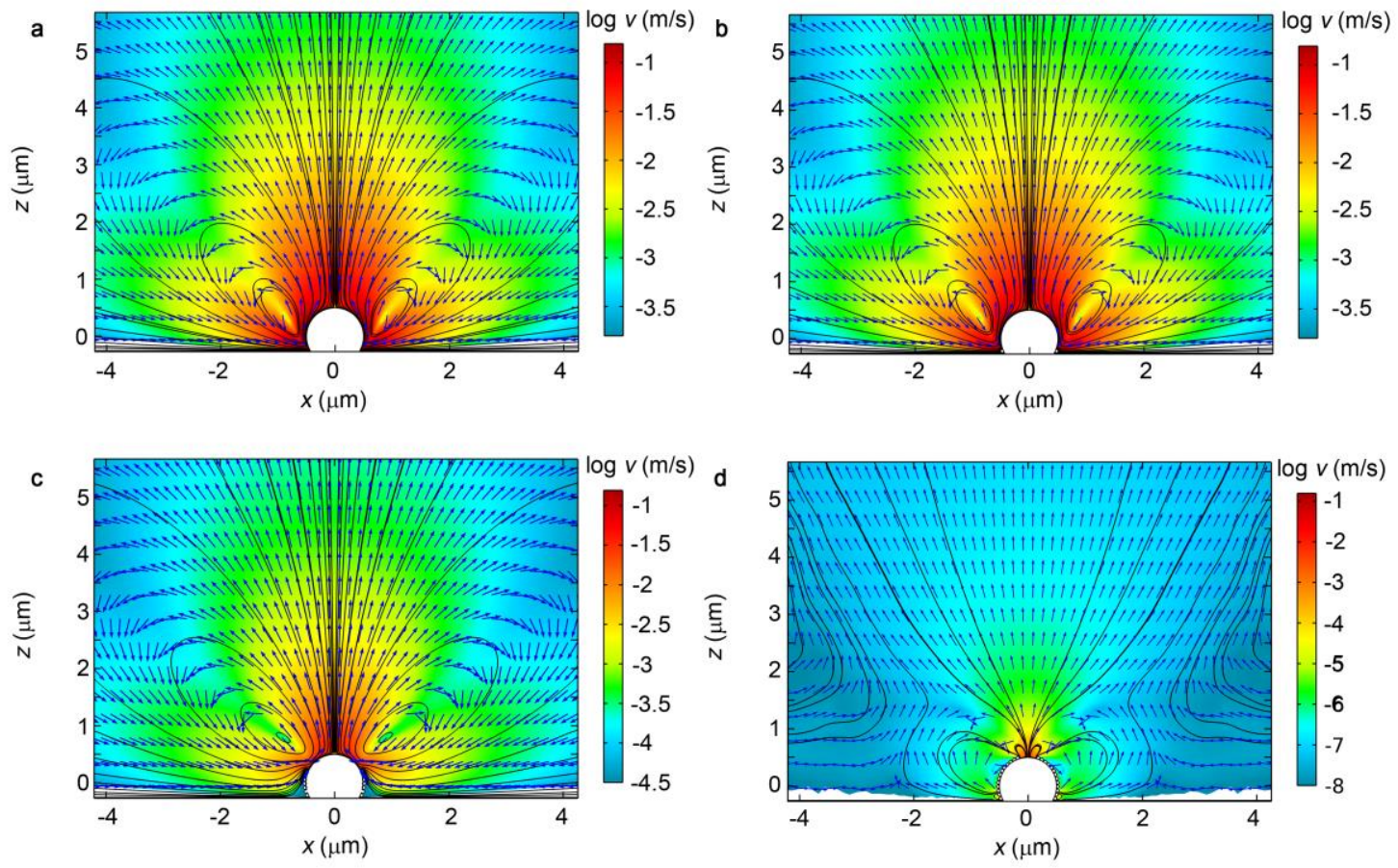

Figure S10. CFD simulations of the flow velocity distribution around a $1 \mu \mathrm{m}$ bubble when $60 \mathrm{~nm}$ PS beads are trapped. The layer number of the $60 \mathrm{~nm}$ PS beads trapped at the gas/liquid interface is (a) 0 , (b) 1 , (c) 7 and (d) 13 . The flow velocity is plotted with logarithmic scale. 

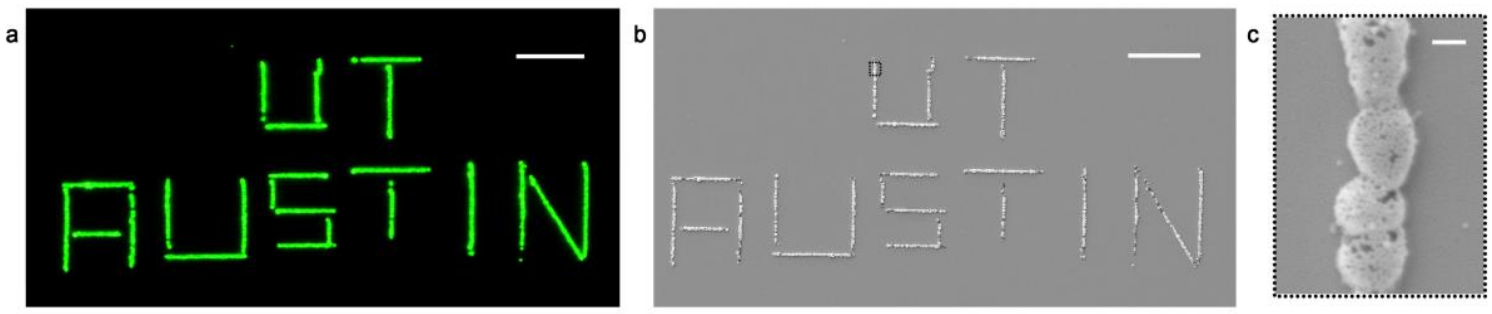

Figure S11. Continuous writing of $60 \mathrm{~nm}$ PS beads into the hollow-ridge patterns. (a) Dark-field optical image and (b) scanning electron micrograph of the hollow-ridge patterns of $60 \mathrm{~nm}$ PS beads in the form of "UT AUSTIN". (c) High-magnification scanning electron micrograph of the small segment as indicated in b, which shows the detailed hollow structure of the $60 \mathrm{~nm}$ PS beads. Scale bar: (a) and (b) $25 \mu \mathrm{m}$; (c) $500 \mathrm{~nm}$.

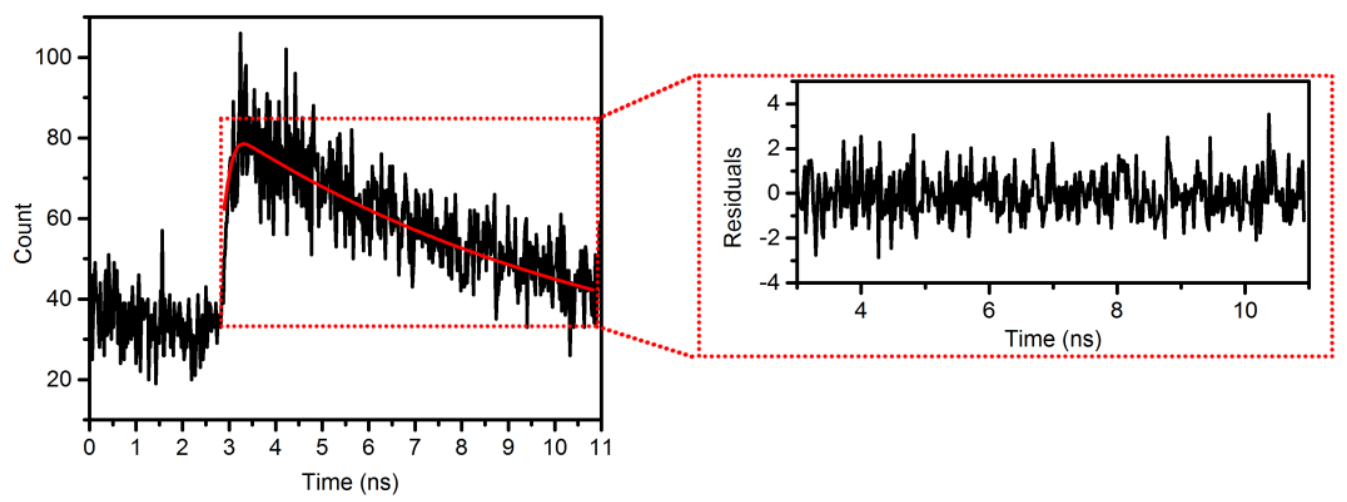

Figure S12. Lifetime measurements for $\mathrm{CdSe} / \mathrm{ZnS}$ quantum dots on the glass substrate. Fitting was performed with the least squares method using a model of a single exponential decay convolved with a Gaussian impulse function. Fitting residuals are shown in the right panel, indicating a good matching between the model and the experimental data. The measured lifetime is $8.1 \mathrm{~ns}$. 